\title{
A method to quantify intracellular glycation in dermal fibroblasts using liquid chromatography coupled to fluorescence detection - Application to the selection of deglycation compounds of dermatological interest
}

\author{
Amandine Andréa,b, Joanna Wdzieczak-Bakalaa ${ }^{a}$ Alexis Kaatio Touréb, Didier Stien ${ }^{c}$ and \\ Véronique Eparvier ${ }^{a}$ \\ ${ }^{a}$ CNRS, Institut de Chimie des Substances Naturelles UPR 2301, Université Paris-Saclay, Gif-Sur- \\ Yvette, France \\ ${ }^{\mathrm{b}}$ Laboratoire Shigeta, Paris, France \\ ${ }^{c}$ CNRS, Laboratoire de Biodiversité et Biotechnologies Microbiennes, Observatoire \\ océanologique, Sorbonne Universités, Banyuls-Sur-Mer, France
}

Corresponding author:

A. Andréa,b, aCNRS, Institut de Chimie des Substances Naturelles UPR 2301, Avenue de la Terrasse, 91190 Gif-Sur-Yvette, France, ${ }^{b}$ Laboratoire Shigeta, 62 boulevard Davout, 75020 Paris, France, amandine.andre@cnrs.fr, 0169823610.

J. Wdzieczak-Bakalaa ${ }^{\text {a }}$ CNRS, Institut de Chimie des Substances Naturelles UPR 2301, Avenue de la Terrasse, 91190 Gif-Sur-Yvette, France, johanna.bakala@cnrs.fr.

K. Touréb, bLaboratoire Shigeta, 62 boulevard Davout, 75020 Paris, France, ktoure@shigeta.fr.

D. Stienc, 'CNRS, Laboratoire de Biodiversité et Biotechnologies Microbiennes, Sorbonne Universités, Observatoire océanologique, 66650 Banyuls-Sur-Mer, France, didier.stien@cnrs.fr.

V. Eparvier ${ }^{\text {a }}$ aCNRS, Institut de Chimie des Substances Naturelles UPR 2301, Avenue de la Terrasse, 91190 Gif-Sur-Yvette, France, veronique.eparvier@cnrs.fr. 


\section{Abstract}

Glycation is a common non-enzymatic reaction between proteins and sugars, which gives rise in the human body to the formation of advanced glycation end products (AGEs). These modifications impacts both extra and intracellular proteins, leading to cells and tissues dysfunctions. In the skin, accumulation of AGEs leads to aesthetic consequences, wrinkles, dark spots and yellowish skin tone, as it can be seen in diabetic patients. Consequently, there is a growing dermatological interest to find compounds able to eliminate AGEs accumulated in skin.

In this context, a method has been developed to detect and quantify intracellular glycation in human dermal fibroblasts. After cultivation of fibroblasts, cell lysates were injected in an HPLC system coupled with a fluorescence detector in by-pass mode. The system allows the simultaneous measurement of global AGEs and particular pentosidine amounts using two sets of wavelengths in a single run of one minute. The immunocytochemistry approach was used to valid the HPLC analysis data.

The method developed was able to quantify changes in global AGEs and pentosidine content in cells in response to glyoxal treatment. Fibroblasts treated with $500 \mu \mathrm{M}$ of glyoxal for 48 hours showed a significant 2.3-fold and 2.6-fold increase in the content of AGEs and pentosidine respectively compared to control cells.

As an application, a screening of natural extracts have been done and the method allowed identifying extracts able to significantly reduce the amount of pentosidine in fibroblasts (- $32 \%)$. These extracts act as deglycation agents of interest in the field of dermatology and cosmetology.

Keywords: Advanced glycation End product (AGEs), HPLC, Fluorescence, Human Dermal Fibroblasts, Immunocytochemistry, Deglycation 


\section{Introduction}

The glycation process, discovered by Maillard in 1912 [1], is a non-enzymatic transformation involving reducing sugars and amino-acids residues of proteins, leading to the formation of a complex and heterogeneous group of compounds named advanced glycation end products (AGEs). More precisely, the process of glycation involves first the reaction of an ose (mainly glucose) with an amine group of a protein amino acid (like lysine or arginine), to form a Schiff base. An Amadori molecular rearrangement then leads to the irreversible formation of AGEs by successive cyclisation and oxidation reactions (Fig. 1.). Due to the various reactions leading to their creation, multiple AGEs have been detected in tissues, and can be divided into three categories: fluorescent AGEs forming reticulations between proteins (for example: pentosidine, crossline), nonfluorescent AGEs forming reticulations between proteins (for example: glyoxal-lysine dimer (GOLD), methylglyoxallysine dimer (MOLD)), and fluorescent or non-fluorescent AGEs forming adducts on proteins (for example: $N$-E-carboxymethyllysine (CML), pyrraline)[2].

AGEs accumulate during the lifetime in whole body including blood plasma, extracellular fluids and cells [3]. Consequently, they are considered as the main causing agent of numerous age-related diseases [4] such as diabetic vascular complications [5], atherosclerosis [6] or Alzheimer's disease [3, 7]. Long lifespan proteins of the body like hemoglobin, collagen or elastin, are known targets of irreversible modifications due to AGEs formation [4]. Extracellular proteins cross-linking leads to cell growth inhibition, impaired cell adhesion and tissue dysfunction [8]. But AGEs are also formed inside cells, causing damages by generation of reactive oxygen species (ROS) [9]. They bond to the 
Receptor of Advanced Glycation End products (RAGE) [10,11], thus increasing

inflammation mediator release via the NF- $\kappa B$ pathway [12]. Activated inflammatory and oxidative cascades lead to the formation of new AGEs in cells. Moreover, excessive ROS levels affect intracellular detoxification systems like the proteasome, as well as other enzymes involved in cellular repairing. All this leads to a decrease in the antioxidative and repair potential of cells [11]. The overall negative effects of AGEs in cells lead to tissue and organ dysfunction [13].

The glycation process does not spare the skin. AGEs have been shown to accumulate in the dermis and epidermis $[14,15]$. Carboxymethyl-lysine (CML) and pentosidine are the most common AGEs in the skin [15]. Aesthetic consequences due to glycation are stiffening of the dermis leading mainly to collapsing of the skin, wrinkles formation, skin tone yellowing and emergence of brown spots, as it can be seen in diabetic patients [16, 17]. Most compounds on the market act by preventing the binding between sugars and proteins, or by reversing the first reversible steps of the glycation reaction. But there are only a few compounds that can reduce the number of final AGEs accumulated in the body [18]. Consequently, there is a growing dermatological, cosmetic and therapeutic interest in finding compounds able to get rid of AGEs accumulated in the skin. However, most of the methods reported in the literature were used to detect and quantify glycation in food $[19,20]$, human urine, plasma or serum [21-24], or in tubo formed AGEs by mixing a single protein with one reducing sugar $[25,26]$. In the skin, studies mainly focus on extracellular proteins modified by glycation $[27,28]$ and not directly on the final AGEs formed at the end of the process. Few methods were reported in the literature to quantify glycation in skin cells, which are suitable with the screening of a very large number of drug candidates. 
The goal of this work was to develop a reliable method able to detect and quantify intracellular AGEs naturally formed and accumulated in human cells upon aging, without using any AGE inducers. The level of AGEs was evaluated by measuring the characteristic autofluorescence of many AGEs [21, 29] and of particular pentosidine [30]. The developed method consist of the analysis of cell lysates using an HPLC system coupled with a fluorescence detector, in by-pass mode to allow a direct measurement of fluorescence using several wavelengths in the same time. The current article provides a detailed description of the technique, and demonstrates how it can be used to screen a large number of natural extracts in order to find new deglycation compounds capable of reducing the intracellular glycation in human fibroblasts.

\section{Material and Methods}

\subsection{Chemical and reagents}

Primary normal human dermal fibroblasts (NHDF) derived from a 54-years-old woman skin tissue were purchased from Promocell and cultured according to the supplier's instructions in fibroblasts growth medium2 (Promocell GmBH, Heidelberg, Germany). Glyoxal solution and phenylmethanesulfonyl fluoride (PMSF) were obtained from Sigma (Sigma-Aldrich, Saint-Quentin Fallavier, France). Radioimmunoprecipitation assay (RIPA) buffer and Phosphate Buffered Solution (PBS) were obtained from ThermoFisher (ThermoFisher Scientific, Villebon sur Yvette, France). TritonX-100 was from Euromedex (Euromedex France, Souffelweyersheim, France). Dimethyl sulfoxide (DMSO) was obtained from Carlo Erba (Carlo Erba Reagents S.A.S, Val de Reuil, France). Tween-20 was obtained from Acros Organics (Acros Organics, Geel, Belgium). Bovine serum albumin (BSA) was obtained from Dutscher (Dominique Dutscher SAS, Brumath, 
France). Paraformaldehyde was obtained from Electron Microscopy Science (EMS, Hartfield, PA, U.S.A). Mouse anti-CarboxyMethyl-Lysine (CML) antibody (KH011) was obtained from Cosmo Bio Co (Cosmo Bio Co LTD, Tokyo, Japan). Goat anti-mouse secondary antibody Alexa-488 conjugate (A11017) was obtained from Lifetechnologies (ThermoFisher Scientific, Villebon sur Yvette, France). Mounting medium with DAPI was obtained from Vector (Vector Laboratories Inc, Burlingame, CA, U.S.A). MilliQ water was obtained using a Millipore Synergy UV-R system (Merck-Millipore, Darmstadt, Germany).

\subsection{Natural extracts}

A library of 269 ethyl acetate extracts of symbiotic microorganisms was used for this study $[31,32]$. Their cytotoxicity have been tested against MRC-5, MDA-MB-435 cell lines. The extracts devoid of toxicity were then tested on primary dermal fibroblasts (NHDF) at three concentrations $(10 \mu \mathrm{g} / \mathrm{mL}, 1 \mu \mathrm{g} / \mathrm{mL}$ and $0,1 \mu \mathrm{g} / \mathrm{mL}$ in DMSO; data available in Table S1 of supplementary material). The extracts having no toxic effect against NHDF at $1 \mu \mathrm{g} / \mathrm{mL}$ were used for the screening of their activity against intracellular AGEs.

\subsection{Cell culture}

Normal human dermal fibroblasts (NHDF) were grown in a humidified atmosphere of 5 $\% \mathrm{CO}_{2}$ at $37^{\circ} \mathrm{C}$ in fibroblasts growth medium 2 .

\subsubsection{Method development}

The NHDF were seeded at the density of 7000 cells $/ \mathrm{cm}^{2}$ on T25 flasks at day 0. 
At day 1, the culture medium was supplemented with glyoxal (final concentration: 250 $\mu \mathrm{M}$ and $500 \mu \mathrm{M}$ ) for 48 hours. The control cells were treated with DMSO only. On day 3, at the end of treatment, culture medium was removed, and cells were washed with HEPES-Buffered saline solution (4-(2-hydroxyethyl)piperazine-1-ethanesulfonic acid) before adding fresh culture medium. Cells were cultivated for another 48 hours before being harvested on day 5 with Trypsine-EDTA solution. Cells were counted using an Automated Cell counter TC20 (BioRad Laboratories, Hercules, CA, U.S.A) and their pellets were kept at $-20^{\circ} \mathrm{C}$ until use. Experiments were done in duplicate.

\subsubsection{Application: Screening of natural extracts}

The NHDF were seeded at the density of 7000 cells $/ \mathrm{cm}^{2}$ on 24 -well-plates at day 0 . At day 1 , the extracts were added in the culture medium (final concentration: $1 \mu \mathrm{g} / \mathrm{mL}$ ) for 24 hours. Each experiment was conducted in duplicate. The control cells were treated with DMSO only. On day 2, after 24 hours of treatment, culture medium was removed, and cells were washed with HEPES-BSS solution before adding fresh culture medium. Cells were cultivated for an additional 48 hours before being harvested on day 4 with a Trypsine-EDTA solution. Cells were counted, freezed and stored at $-20^{\circ} \mathrm{C}$ until use.

\subsection{Cell lysis}

Cell pellets were lysed for 30 minutes on ice, using RIPA lysis buffer in which a PMSF solution was added to prevent the action of proteases. After centrifugation at $14000 \mathrm{~g}$, the supernatants were stored at $-20^{\circ} \mathrm{C}$ until analysis.

\subsection{HPLC/Fluorescence method:}




\subsubsection{Sample preparation for HPLC/fluorescence}

The day of the analysis, cell lysates were diluted three times with PBS 1X, filtered, and put into an HPLC insert vial. HPLC vials were kept at $4^{\circ} \mathrm{C}$ within the HPLC autosampler during all the experiment.

\subsubsection{HPLC/Fluorescence analysis}

For the analysis of the cell lysates, a Waters 2690/S separation module HPLC system coupled with a Waters W2475 fluorescence detector was used (Waters Corporation, Guyancourt, France). Since no separation was required, a by-pass was set-up instead of the column. Wavelengths on the fluorescence detector were set at $\lambda_{\mathrm{ex}}=370 \mathrm{~nm} / \lambda \mathrm{em}=$ $445 \mathrm{~nm}$ for measuring global AGEs amount, and at $\lambda_{\mathrm{ex}}=335 \mathrm{~nm} / \lambda_{\mathrm{em}}=385 \mathrm{~nm}$ for measuring particular pentosidine amount, as previously described [21, 29, 30]. Mobile phase used was $100 \%$ milliQ $\mathrm{H}_{2} \mathrm{O}$ at a flow rate of $0.5 \mathrm{~mL} / \mathrm{min}$. Run length was 1 minute. Cell lysates $(6 \mu \mathrm{L})$ were injected in triplicate, and a blank (water) was injected every 8 runs to confirm that no fluorescence accumulated in pipes.

\subsubsection{Results analysis}

Fluorescence picks were automatically integrated using Empower software (Waters Corporation, Guyancourt, France). Picks areas were analyzed and correspond to the cell lysate fluorescence value expressed in arbitrary units.

To allow the comparison between treatments, the fluorescence values were normalized for 1000 cells.

\subsection{CML immunofluorescence}


NHDF were plated on glass coverslips set into a 24-wells-plate and cultured in standard conditions as described above. On day 4 , the fibroblasts were fixed with $2 \%$ paraformaldehyde (500 $\mu \mathrm{L}$ ) for 12 minutes at room temperature, permeabilized with $0.2 \%$ TritonX-100 (500 $\mu \mathrm{L})$ for 10 minutes and finally blocked with $5 \%$ BSA, $0.2 \%$ Tween-20 (500 $\mu \mathrm{L})$ for further 10 minutes. Cells were then incubated with the primary antibody anti-CML (mouse) at a concentration of $7 \mu \mathrm{g} / \mathrm{mL}(30 \mu \mathrm{L})$ at $4{ }^{\circ} \mathrm{C}$ overnight, followed by incubation with Alexa-488 anti-mouse secondary antibody diluted at 1:200 $(30 \mu \mathrm{L})$ for 2 hours at room temperature. After washing with $5 \%$ BSA, $0.2 \%$ Tween-20 (3x500 $\mu \mathrm{L})$, coverslips were mounted onto microscope slides using a DAPI containing mounting medium, and observed under a confocal photonic microscope (Spinning-Disk microscope, Roper/Nikon - parameters for DAPI: Laser 447/60 405 ; Gain : 3(4x) ; Digitizer : 5MHz ; EM gain : 150 ; Exposition : 200 ms ; Laser power : 30\% - parameters for Alexa 488 : Laser 525/45 491 ; Gain : 3 (4x) ; Digitizer : 5MHz ; EM gain : 200 ; Exposition : 300ms ; Laser power: 25\%). Images are recorded using a Z stack, allowing fluorescence to be recorded at different positions in the cell. Five images were recorded by coverslips (ten pictures per treatment condition). All pictures were taken with the same microscope parameters. Fluorescence quantification on the images was done using Image J software.

\subsection{Statistical analysis}

Data were presented as mean \pm standard error of the mean. The mean data were analyzed with one-way analysis of variance (one way ANOVA) followed by Dunnett's multiple comparison test. A P $<0.05$ was considered to be significant.

\subsection{Method validation}


To determine the reproducibility of the method, we calculated the relative standard deviation (RSD) for triplicates of the same sample, and biological duplicates of the same treatment (data available in Table S2 of supplementary material).

\section{Results}

\subsection{Method development}

We first examined whether the proposed approach was able to detect and quantify the variations in the amount of AGEs and pentosidine in lysates of fibroblasts treated with $250 \mu \mathrm{M}$ and $500 \mu \mathrm{M}$ of glyoxal for 48 hours.

Glyoxal is a dicarbonyl formed in vivo mainly by auto-oxidation of reducing sugars and by lipid peroxidation. Both highly reactive carbonyl groups of glyoxal react with the amino groups of proteins to form AGEs as previously reported [33-35].

Lysates of glyoxal treated cells and lysates of untreated controls were injected in triplicate in the HPLC system in by-pass mode, using MilliQ $\mathrm{H}_{2} \mathrm{O}$ as mobile phase, and the signal was recorded with a fluorescence detector using two sets of wavelengths for the simultaneous detection of AGEs $\left(\lambda_{\mathrm{ex}}=370 \mathrm{~nm} / \lambda_{\mathrm{em}}=445 \mathrm{~nm}\right)$ and pentosidine $\left(\lambda_{\mathrm{ex}}\right.$ $=335 \mathrm{~nm} / \lambda_{\mathrm{em}}=385 \mathrm{~nm}$ ). The use of the HPLC system allows the analysis of small amounts of samples, with good reproducibility. The fluorescence spectra of pentosidine recorded in control cells lysate (triplicate) are presented in Figure 2. The fluorescence value corresponds to peak integration value. To allow a reliable comparison between the treatments, the number of cells harvested at the end of the cell culture was counted, and the fluorescence values were normalized for 1000 cells.

As shown in Table I, the treatment of fibroblasts with glyoxal increases significantly the fluorescence detected in cell lysates for both AGEs and pentosidine, in a dose-dependent 
manner. A 1.3-fold and 2.2-fold increase in the amount of intracellular AGEs present in cells treated respectively with $250 \mu \mathrm{M}$ and $500 \mu \mathrm{M}$ of glyoxal for 48 hours, compared to controls was observed. For pentosidine, a 1.4-fold and 2.6-fold increase in cells treated respectively with $250 \mu \mathrm{M}$ and $500 \mu \mathrm{M}$ of glyoxal was detected.

To valid these findings, we analyzed the level of carboxymethyl-lysine (CML) by immunocytochemistry in cells treated with $500 \mu \mathrm{M}$ of glyoxal for 48 hours (Fig. 3.). CML is an AGE forming adducts on proteins. Quantification of the immunofluorescence signal on images taken by confocal photonic microscopy shows that the intracellular CML content was indeed significantly higher in cells treated with glyoxal $(1.57 \pm 0.07$ increase compared to non-treated control cells).

\subsection{Application: The effect of natural products on the amount of intracellular pentosidine}

As the developed method was able to detect variations in the content of AGEs in cells treated with different concentrations of glyoxal, we used it to screen eighty natural extracts and evaluate their potential to reduce the quantity of intracellular AGEs and pentosidine in human skin cells. Treatment of fibroblasts with extracts was done for 24 hours, in biological duplicate. The intracellular AGEs and pentosidine level was recorded by HPLC with fluorescence detector as described above. Aminoguanidine, an AGE inhibitor compound, was chosen as control. Table II show the results of twentythree of the extracts as the mean amount of pentosidine in cells compared to control.

The screening conducted in biological duplicates showed that aminoguanidine did not reduce the intracellular amount of pentosidine. However, among the eighty natural extracts, seven of them were shown to significantly reduce the intracellular content of 
pentosidine in dermal fibroblasts. SNB-GTC2701 was the most active extract, reducing intracellular pentosidine content by $32 \%$ in fibroblasts after 24 hours of treatment at 1 $\mu \mathrm{g} / \mathrm{mL}$.

\subsection{Reproducibility}

The reproducibility of the method was evaluated taking into account the triplicates of the same sample and biological duplicates of the same treatment.

The relative standard deviations of samples (RSD) between the triplicates of the same sample ranged within $10 \%$ (except for treatment SNB-CN76) for AGEs and for pentosidine (Table S2). RSD calculated between biological duplicates showed good reproducibility as well $(<10 \%)$. According to these results, the method can be considered as robust.

\section{Discussion}

The present method was developed to quantify the amount of intracellular AGEs and pentosidine in human dermal fibroblasts lysates, using their characteristic autofluorescent properties.

To develop the method, we used glyoxal treated fibroblasts. Glyoxal is mainly produced during the autooxidation of reducing sugars, and is a source of macromolecular damage in cells. Treatment of fibroblasts with glyoxal was shown to induce a senescent phenotype in vitro, and to lead to an increase of AGEs levels, thus providing a model of accelerated cellular aging [33].

Results obtained with the developed method confirmed that glyoxal induces a change in the AGE content in fibroblasts. Moreover, the HPLC-fluorescent method was efficient to detect and quantify these changes. 
Besides, by analyzing the results obtained during the development phase, we noticed that the glycation level of the non-treated dermal fibroblasts obtained from a 54 years old donor was high enough to be easily detected and quantified by the HPLC coupled with fluorescence method. In fact, AGEs accumulates in vivo during normal aging, and the detection of naturally occurring AGEs in fibroblasts with our method was of high interest for the next step of our project. This allowed us to screen the natural extracts library on cells that have not undergone glyoxal treatment, thus enabling the screening to be performed under conditions closest to the biological conditions in vivo. During the screening, aminoguanidine was chosen as a control compound. This molecule works as a scavenger of reactive dicarbonyl intermediates in the Maillard reaction, therefore inhibiting AGE formation. Our results showed that aminoguanidine did not reduce the pentodisine level in fibroblasts. This finding is in line with published data on its mode of action preventing the condensation of reducing sugars with aminoacids residues, or inhibiting the first reversible reaction of the glycation process [36]. We provide new evidence that aminoguanidine is not able to target AGEs at their final stage.

Among eighty natural extracts tested, seven of them showed positive results on pentosidine, significantly reducing its content in dermal fibroblasts after 24 hours of treatment. Comparing these results with those of aminoguanidine, we hypothesize that the mechanism of action of the tested active extracts might be linked to a possible activation or inhibition of specific biological pathways. Literature shows that AGE receptor (AGER) subunit AGER-1 (OST-48) binds to AGEs, triggering endocytosis and elimination of AGEs by cells, while this receptor does not bind to early glycation products like Amadori products [37-39]. Pentosidine can be degraded through this mechanism. We observed by western blot using a specific AGER-1 antibody that AGER-1 
was overexpressed in cells treated with three of the active extracts (Figure S1).

Although further investigations will be necessary, it is possible that the active extracts trigger a natural cell detoxification mechanism.

Due to the dermatological interest of finding compounds able to decrease the amount of formed AGEs in skin, an effort was made to make the assay more rapid and as simple as possible, allowing the screening of a large number of candidates in one time.

The screening of a large number of substances in a biological test requires cultivating cells in large amounts. But miniaturization of biological tests into multiwall plates lower the available quantity of samples to be analyzed. The main advantage of using an HPLC system is to benefit from the auto sampler allowing precise sampling of small amounts like few $\mu \mathrm{L}$. This allowed us to miniaturize our biological screening into 24-wells plates, and so to decrease the amount of cells to be cultivated as well as the extract quantity to be applied as treatment.

\section{Conclusion}

The method described in this article allows detecting and quantifying intracellular levels of AGEs in human dermal fibroblasts. It is reliable, reproducible, simple and can be performed at rather low-cost. The use of HPLC coupled to fluorescence detection has many advantages. First, the fluorescence detection at two wavelengths at the same time reduces the quantity of sample required for analysis ( $6 \mu \mathrm{L}$ per injections), allowing culture of cells in 24-wells plates. Then, once the cells harvested, one requires only a few steps of sample preparation. Finally, the automatic injection of the samples in a short one-minute run, with good reproducibility between technical and biological replicates, makes it suitable for the rapid and reliable screening of a large number of samples. 
Contrary to commonly used methods i.e. in tubo tests employing one reducing sugar and one protein or methods targeting extracellular proteins modified by glycation, our experimental approach allows detection and quantification of intracellular AGEs formed and accumulated during aging in cells without induction of glycation.

Eventually, the method was applied to test natural extracts and revealed the potential of some of them to reduce the amount of AGEs naturally accumulated in cells with aging, thus suggesting that new deglycation compounds of dermatological and cosmetic interest may be discovered. The mode of action of the active extracts needs to be further characterized but seems to involve complex biological reactions. Thus, the molecules responsible for the activity will be characterized after bioguided isolation.

\section{Aknowledgements}

The present work has benefited from the facilities and expertise of the HPLC facilities of CNRS-ICSN. We shall address our appreciation to Odile Thoison and Frank Pelissier for their technical assistance.

The present work has benefited from the facilities and expertise of the light microscopy facilities of Imagerie-Gif. This core facility is member of the Infrastructures en Biologie Santé et Agronomie (IBiSA), and is supported by the French National Research Agency under Investments for the Future programs "France-BioImaging", and the Labex "Saclay Plant Science" (ANR-10-INSB-04-01 and ANR-11-IDEX-0003-02, respectively). A special thanks to Romain Le Bars and Laëtitia Besse for their invaluable help during the microscopy sessions. 


\section{Funding}

This work has benefited from a CIFRE grant managed by the Association Nationale de la

Recherche et de la Technologie, France (ANRT, $\left.\mathrm{n}^{\circ} 2013-1326\right)$, and from an

"Investissement d'Avenir" grant managed by Agence Nationale de la Recherche, France

(CEBA, ref. ANR-10-LABX-25-01).

\section{Conflict of Interest}

The authors declare that they have no conflict of interest.

\section{REFERENCES}

1. Maillard, L. Action des acides aminés sur les sucres, formation des mélanoïdines par voie méthodique. C R Acad Sci. (154):66-8 (1912).

2. Ahmed, N. Advanced glycation endproducts-role in pathology of diabetic complications. Diabetes Res Clin Prat. 67(1):3-21 (2005).

3. Vlassara, H. Advanced glycation in health and disease: role of the modern environment. Ann NY Acad Sci. 1043:452-60 (2005).

4. Kasper, M. and Funk, R.H. Age-related changes in cells and tissues due to advanced glycation end products (AGEs). Arch Gerontol Geriatr. 32(3):233-43 (2001).

5. Goldin, A., Beckman, J.A., Schmidt, A.M. and Creager, M.A. Advanced glycation end products: sparking the development of diabetic vascular injury. Circulation. 114(6):597-605 (2006).

6. Kume, S., Takeya, M., Mori, T., Araki, N., Suzuki, H., Horiuchi, S., et al. Immunohistochemical and ultrastructural detection of advanced glycation end products in atherosclerotic lesions of human aorta with a novel specific monoclonal antibody. Am J Pathol. 147(3):654-67 (1995).

7. Baynes, J.W. The role of AGEs in aging: causation or correlation. Exp Gerontol. 36(9):1527-37 (2001).

8. Paul, R.G. and Bailey, A.J. The effect of advanced glycation end-product formation upon cellmatrix interactions. Int J Biochem Cell Biol. 31(6):653-60 (1999).

9. Valencia, J.V., Weldon, S.C., Quinn, D., Kiers, G.H., DeGroot, J., TeKoppele, J.M., et al. Advanced glycation end product ligands for the receptor for advanced glycation end products: biochemical characterization and formation kinetics. Anal Biochem. 324(1):68-78 (2004).

10. Liu, Y., Liang, C., Liu, X., Liao, B., Pan, X., Ren, Y., et al. AGEs increased migration and inflammatory responses of adventitial fibroblasts via RAGE, MAPK and NF-kappaB pathways. Atherosclerosis. 208(1):34-42 (2010).

11. Ott, C., Jacobs, K., Haucke, E., Navarrete Santos, A., Grune, T. and Simm, A. Role of advanced glycation end products in cellular signaling. Redox Biol. 2:411-29 (2014).

12. Kasper, M., Schinzel, R., Niwa, T., Munch, G., Witt, M., Fehrenbach, H., et al. Experimental induction of AGEs in fetal L132 lung cells changes the level of intracellular cathepsin D. Biochem Biophys Res Commun. 261(1):175-82 (1999).

13. Badenhorst, D., Maseko, M., Tsotetsi, O.J., Naidoo, A., Brooksbank, R., Norton, G.R., et al. Crosslinking influences the impact of quantitative changes in myocardial collagen on cardiac stiffness and remodelling in hypertension in rats. Cardiovasc Res. 57(3):632-41 (2003).

14. Kawabata, K., Yoshikawa, H., Saruwatari, K., Akazawa, Y., Inoue, T., Kuze, T., et al. The presence of N(epsilon)-(Carboxymethyl) lysine in the human epidermis. Biochim Biophys Acta. 1814(10):1246-52 (2011). 
15. Gkogkolou, P. and Bohm, M. Advanced glycation end products: Key players in skin aging? Dermatoendocrinol. 4(3):259-70 (2012).

16. Lee, E.J., Kim, J.Y. and Oh, S.H. Advanced glycation end products (AGEs) promote melanogenesis through receptor for AGEs. Sci Rep. 6:27848 (2016).

17. Dyer, D.G., Dunn, J.A., Thorpe, S.R., Bailie, K.E., Lyons, T.J., McCance, D.R., et al. Accumulation of Maillard reaction products in skin collagen in diabetes and aging. J Clin Invest. 91(6):2463-9 (1993).

18. Yang, S., Litchfield, J.E. and Baynes, J.W. AGE-breakers cleave model compounds, but do not break Maillard crosslinks in skin and tail collagen from diabetic rats. Arch Biochem Biophys. 412(1):42-6 (2003).

19. Tareke, E., Forslund, A., Lindh, C.H., Fahlgren, C. and Ostman, E. Isotope dilution ESI-LC-MS/MS for quantification of free and total Nepsilon-(1-Carboxymethyl)-L-Lysine and free Nepsilon-(1Carboxyethyl)-L-Lysine: comparison of total Nepsilon-(1-Carboxymethyl)-L-Lysine levels measured with new method to ELISA assay in gruel samples. Food Chem. 141(4):4253-9 (2013).

20. He, J., Zeng, M., Zheng, Z., He, Z. and Chen, J. Simultaneous determination of Ne-(carboxymethyl) lysine and Ne-(carboxyethyl) lysine in cereal foods by LC-MS/MS. Eur Food Res Technol. (238):367-74 (2014).

21. Munch, G., Keis, R., Wessels, A., Riederer, P., Bahner, U., Heidland, A., et al. Determination of advanced glycation end products in serum by fluorescence spectroscopy and competitive ELISA. Eur J Clin Chem Clin Biochem. 35(9):669-77 (1997).

22. Hanssen, N.M., Engelen, L., Ferreira, I., Scheijen, J.L., Huijberts, M.S., van Greevenbroek, M.M., et al. Plasma levels of advanced glycation endproducts Nepsilon-(carboxymethyl)lysine, Nepsilon(carboxyethyl)lysine, and pentosidine are not independently associated with cardiovascular disease in individuals with or without type 2 diabetes: the Hoorn and CODAM studies. J Clin Endocrinol Metab. 98(8):E1369-73 (2013).

23. Price, D.L., Rhett, P.M., Thorpe, S.R. and Baynes, J.W. Chelating activity of advanced glycation endproduct inhibitors. J Biol Chem. 276(52):48967-72 (2001).

24. De La Maza, M.P., Bravo, A., Leiva, L., GattÁS, V., Petermann, M., Garrido, F., et al. Fluorescent serum and urinary advanced glycoxidation end-products in non- diabetic subjects. Biological Research. 40:203-12 (2007).

25. Ahmed, N., Argirov, O.K., Minhas, H.S., Cordeiro, C.A. and Thornalley, P.J. Assay of advanced glycation endproducts (AGEs): surveying AGEs by chromatographic assay with derivatization by 6aminoquinolyl-N-hydroxysuccinimidyl-carbamate and application to Nepsilon-carboxymethyl-lysine- and Nepsilon-(1-carboxyethyl)lysine-modified albumin. Biochem J. 364(1):1-14 (2002).

26. Schmitt, A., Schmitt, J., Munch, G. and Gasic-Milencovic, J. Characterization of advanced glycation end products for biochemical studies: side chain modifications and fluorescence characteristics. Anal Biochem. 338(2):201-15 (2005).

27. Gasser, P., Arnold, F., Peno-Mazzarino, L., Bouzoud, D., Luu, M.T., Lati, E., et al. Glycation induction and antiglycation activity of skin care ingredients on living human skin explants. Int J Cosmet Sci. 33(4):366-70 (2011).

28. Kobayashi, K. and Igimi, H. Glycation index of hair for non-invasive estimation of diabetic control. Biol Pharm Bull. 19(4):487-90 (1996).

29. Galler, A., Muller, G., Schinzel, R., Kratzsch, J., Kiess, W. and Munch, G. Impact of metabolic control and serum lipids on the concentration of advanced glycation end products in the serum of children and adolescents with type 1 diabetes, as determined by fluorescence spectroscopy and nepsilon(carboxymethyl)lysine ELISA. Diabetes Care. 26(9):2609-15 (2003).

30. Odetti, P., Fogarty, J., Sell, D.R. and Monnier, V.M. Chromatographic quantitation of plasma and erythrocyte pentosidine in diabetic and uremic subjects. Diabetes. 41(2):153-9 (1992).

31. Casella, T.M., Eparvier, V., Mandavid, H., Bendelac, A., Odonne, G., Dayan, L., et al. Antimicrobial and cytotoxic secondary metabolites from tropical leaf endophytes: Isolation of antibacterial agent pyrrocidine C from Lewia infectoria SNB-GTC2402. Phytochemistry. 96:370-7 (2013).

32. Nirma, C., Eparvier, V. and Stien, D. Antifungal Agents from Pseudallescheria boydii SNB-CN73 Isolated from a Nasutitermes sp. Termite. J Nat Prod. 76(5):988-91 (2013).

33. Sejersen, H. and Rattan, S.I. Dicarbonyl-induced accelerated aging in vitro in human skin fibroblasts. Biogerontology. 10(2):203-11 (2009).

34. Bulteau, A.L., Verbeke, P., Petropoulos, I., Chaffotte, A.F. and Friguet, B. Proteasome inhibition in glyoxal-treated fibroblasts and resistance of glycated glucose-6-phosphate dehydrogenase to $20 \mathrm{~S}$ proteasome degradation in vitro. J Biol Chem. 276(49):45662-8 (2001). 
35. Ahmed, M.U., Brinkmann Frye, E., Degenhardt, T.P., Thorpe, S.R. and Baynes, J.W. N-epsilon(carboxyethyl)lysine, a product of the chemical modification of proteins by methylglyoxal, increases with age in human lens proteins. Biochem J. 324(2):565-70 (1997).

36. Nagai, R., Murray, D.B., Metz, T.O. and Baynes, J.W. Chelation: A Fundamental Mechanism of Action of AGE Inhibitors, AGE Breakers, and Other Inhibitors of Diabetes Complications. Diabetes. 61(3):549-59 (2012).

37. Li, Y.M., Mitsuhashi, T., Wojciechowicz, D., Shimizu, N., Li, J., Stitt, A., et al. Molecular identity and cellular distribution of advanced glycation endproduct receptors: relationship of p60 to OST-48 and p90 to 80K-H membrane proteins. Proc Natl Acad Sci U S A. 93(20):11047-52 (1996).

38. Lu, C., He, J.C., Cai, W., Liu, H., Zhu, L. and Vlassara, H. Advanced glycation endproduct (AGE) receptor 1 is a negative regulator of the inflammatory response to AGE in mesangial cells. Proc Natl Acad Sci U S A. 101(32):11767-72 (2004).

39. Yang, Z., Makita, Z., Horii, Y., Brunelle, S., Cerami, A., Sehajpal, P., et al. Two novel rat liver membrane proteins that bind advanced glycosylation endproducts: relationship to macrophage receptor for glucose-modified proteins. JExp Med.174(3):515 (1991). 
Legends to figures:

Figure 1: Glycation reaction of proteins with glucose leading to the formation of AGEs, and structure of Pentosidine crosslink.

Figure 2: Example of fluorescence signal recorded for pentosidine $\left(\lambda_{\mathrm{ex}}=335 \mathrm{~nm} / \lambda_{\mathrm{em}}=\right.$ $385 \mathrm{~nm}$ ) in lysate of control cells. Injections were done in triplicate. Peak integration (area) was done with Empower software (Waters).

Figure 3: Confocal microscopy images (X40) of human dermal fibroblasts treated for 48 hours with (a) DMSO as control and (b) $500 \mu \mathrm{M}$ of glyoxal. Nucleus labeling by DAPI (blue) ; Immunological labeling of CML by Alexa 488 (green).

Tables:

Table I. AGEs $\left(\lambda_{\text {ex }}=370 \mathrm{~nm} / \lambda_{\mathrm{em}}=445 \mathrm{~nm}\right)$ and pentosidine expression $\left(\lambda_{\mathrm{ex}}=335 \mathrm{~nm} /\right.$ $\lambda_{\mathrm{em}}=385 \mathrm{~nm}$ ) in NHDF treated with $250 \mu \mathrm{M}$ and $500 \mu \mathrm{M}$ glyoxal for 48 hours.

Concentrations have been normalized relatively to the negative control, for 1000 cells. Significance levels when compared to negative control were calculated by one-way ANOVA followed by Dunett's multiple comparison test ${ }^{* * *} \mathrm{P}<0.001$ compared to control.

\begin{tabular}{lll}
\hline \hline Treatment & AGEs expression \pm SD & Pentosidine \pm SD \\
\hline \hline GO $250 \mu \mathrm{M}$ & $1.32 \pm 0.16^{* * *}$ & $1.38 \pm 0.03^{* * *}$ \\
GO $500 \mu \mathrm{M}$ & $2.16 \pm 0.07^{* * *}$ & $2.61 \pm 0.02^{* * *}$ \\
\hline \hline
\end{tabular}


Table II. Effect of 23 different microbial extracts and aminoguanidine on pentosidine expression compared to control, measured by the HPLC/fluorescence method in human dermal fibroblasts. Concentrations have been normalized relatively to the negative control, for 1000 cells. Significance levels when compared to negative control were calculated by one-way ANOVA followed by Dunett's multiple comparison test; ${ }^{*} \mathrm{P}<0.05$; ${ }^{* *} \mathrm{P}<0.01 ;{ }^{* * *} \mathrm{P}<0.001$ compared to control, $\mathrm{ns}=$ not significant.

\begin{tabular}{ll}
\hline \hline Treatment & Pentosidine amount \pm SD \\
\hline Control (untreated) & $1.00 \pm 0.05$ \\
Aminoguanidine & $1.13 \pm 0.18^{\mathrm{ns}}$ \\
\hline \hline SNB-GTC2701 & $0.68 \pm 0.07^{* * *}$ \\
SNB-CN20 & $0.71 \pm 0.01^{* *}$ \\
SNB-CN102 & $0.76 \pm 0.03^{* *}$ \\
SNB-CN55 & $0.79 \pm 0.01^{* *}$ \\
SNB-CN76 & $0.80 \pm 0.03^{* * *}$ \\
SNB-GSS07 & $0.85 \pm 0.04^{\mathrm{ns}}$ \\
SNB-CN54 & $0.87 \pm 0.05^{*}$ \\
SNB-CN79 & $0.87 \pm 0.07^{\mathrm{ns}}$ \\
SNB-GSS04 & $0.88 \pm 0.01^{* *}$ \\
SNB-CN13 & $0.89 \pm 0.09^{\mathrm{ns}}$ \\
SNB-CN59 & $0.89 \pm 0.04^{\mathrm{ns}}$ \\
SNB-GTC2810 & $0.91 \pm 0.10^{\mathrm{ns}}$ \\
SNB-CN104 & $0.93 \pm 0.04^{\mathrm{ns}}$ \\
SNB-CN66 & $0.93 \pm 0.01^{\mathrm{ns}}$ \\
SNB-CN27 & $0.94 \pm 0.10^{\mathrm{ns}}$ \\
SNB-CN69 & $0.97 \pm 0.11^{\mathrm{ns}}$ \\
SNB-CN67 & $0.97 \pm 0.05^{\mathrm{ns}}$ \\
SNB-CN75 & $0.98 \pm 0.05^{\mathrm{ns}}$ \\
SNB-LD 9.2 & $1.02 \pm 0.10^{\mathrm{ns}}$ \\
SNB-CN98 & $1.14 \pm 0.03^{\mathrm{ns}}$ \\
SNB-CN1 & $1.16 \pm 0.09^{*}$ \\
SNB-CN85 & $1.18 \pm 0.04^{\mathrm{ns}}$ \\
SNB-CN10 & $1.58 \pm 0.27^{* *}$ \\
\hline \hline
\end{tabular}



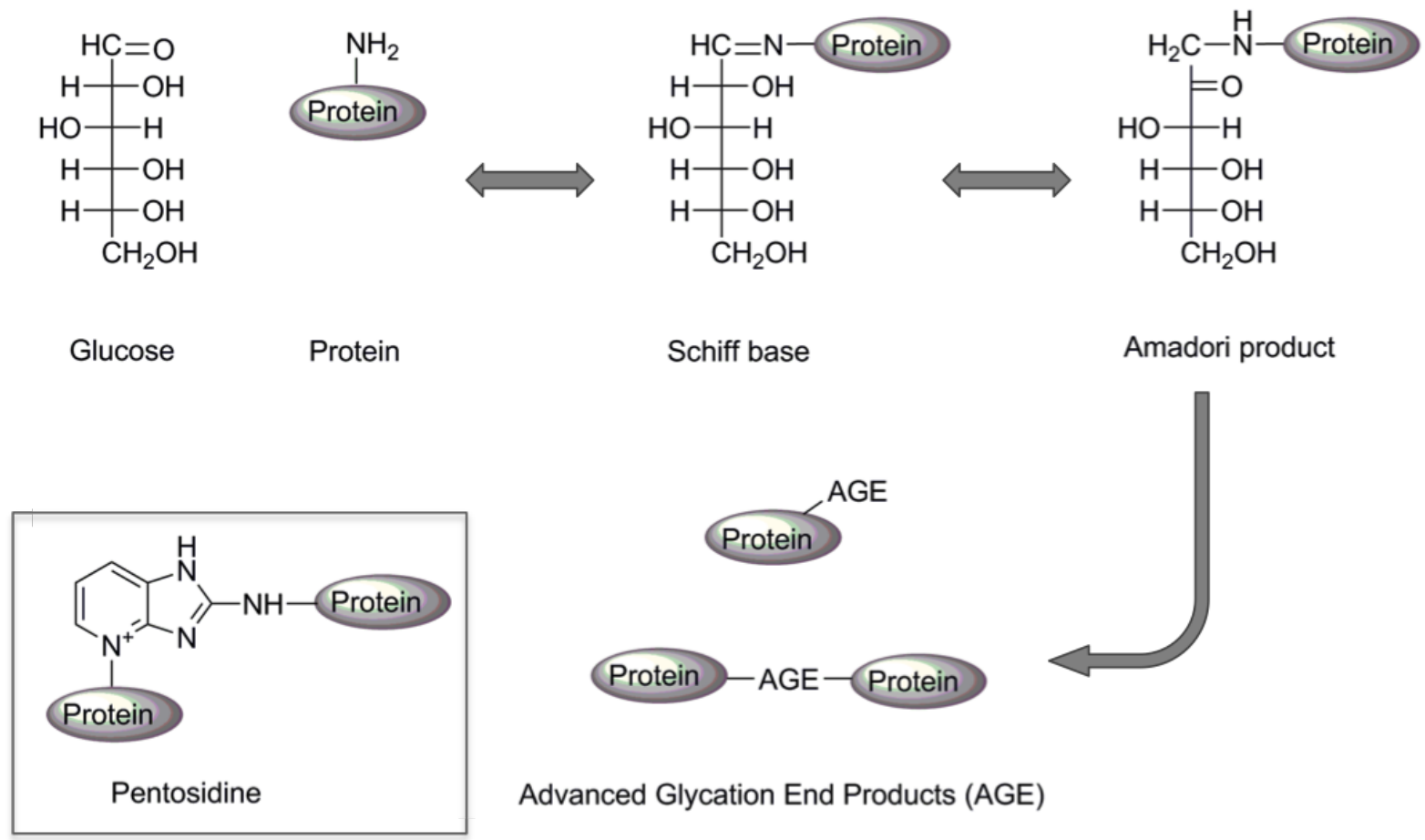

Glucose

Protein

Schiff base

Amadori product

Figure 1._Glycation reaction of proteins with glucose leading to the formation of AGEs, and structure of Pentosidine crosslink.
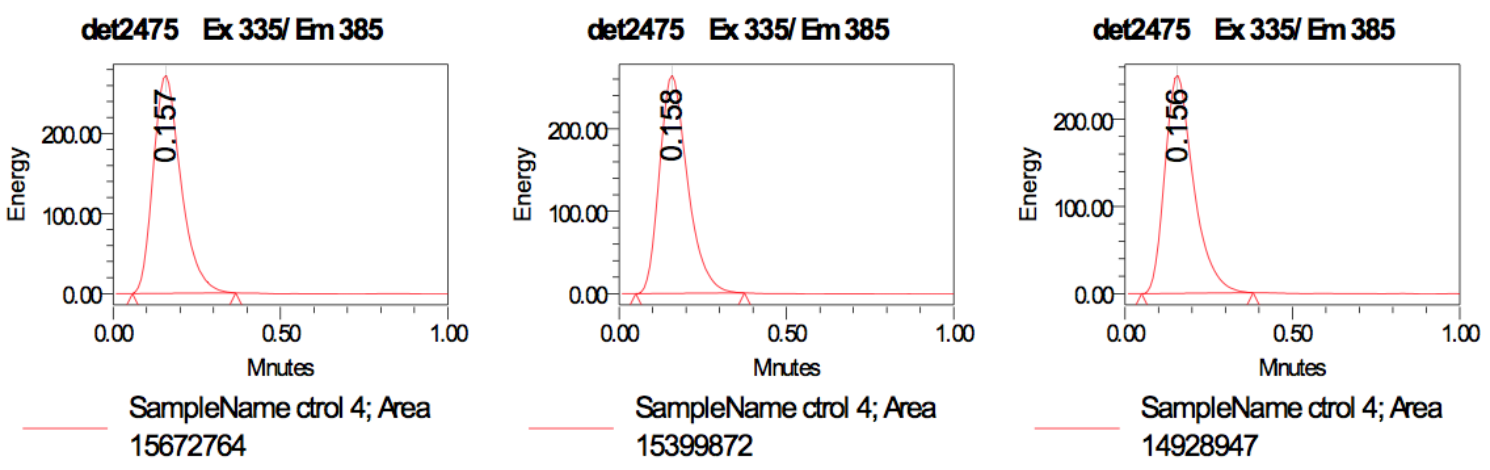

Figure 2. Example of fluorescence signal recorded for pentosidine $\left(\lambda_{\mathrm{ex}}=335 \mathrm{~nm} / \lambda_{\mathrm{em}}=\right.$ $385 \mathrm{~nm}$ ) in lysate of control cells. Injections were done in triplicate. Peak integration (area) was done with Empower software (Waters). 


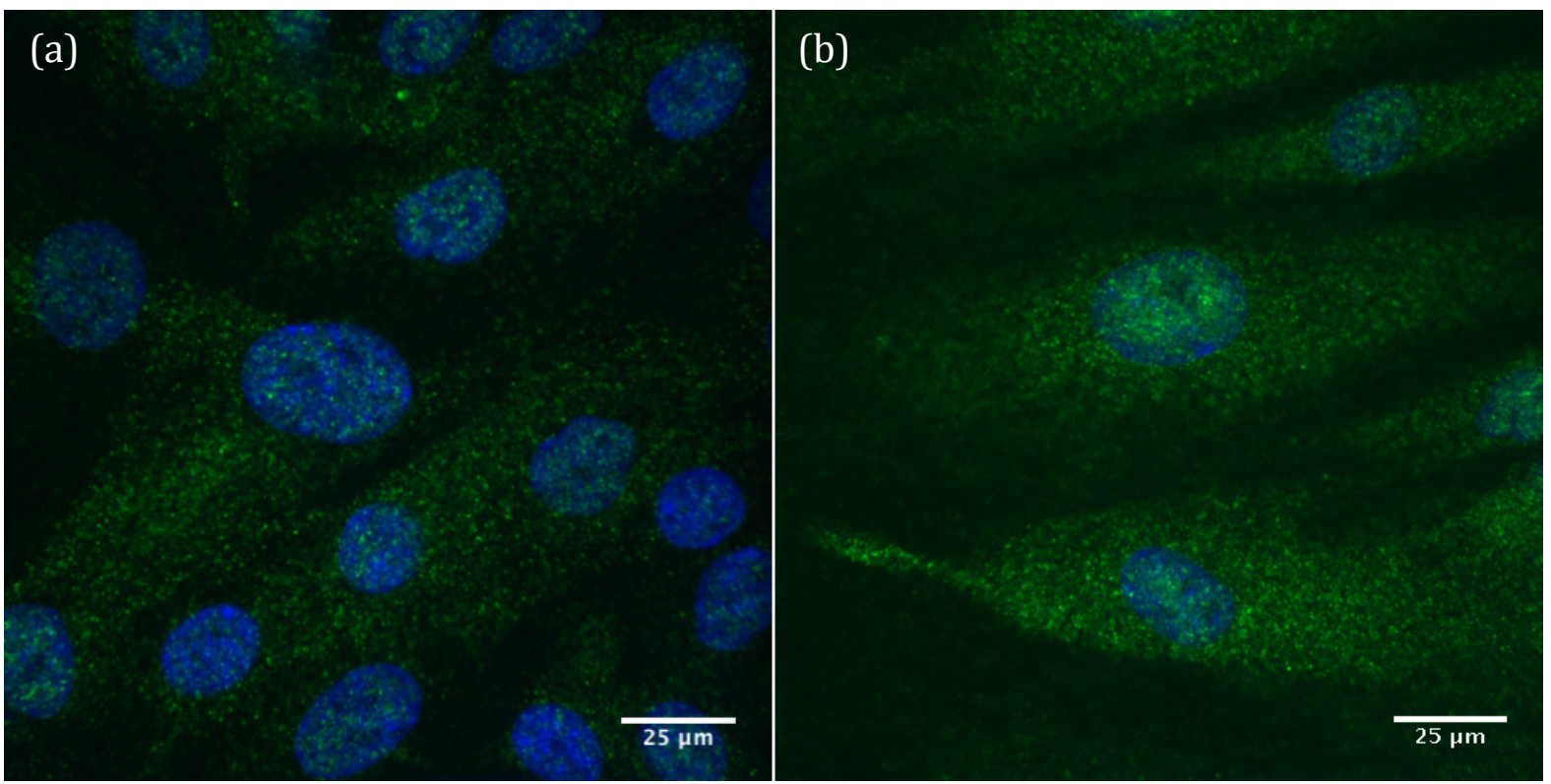

Figure 3. Confocal microscopy images (X40) of human dermal fibroblasts treated for 48 hours with (a) DMSO as control and (b) $500 \mu \mathrm{M}$ of glyoxal. Nucleus labeling by DAPI (blue) ; Immunological labeling of CML by Alexa 488 (green). 


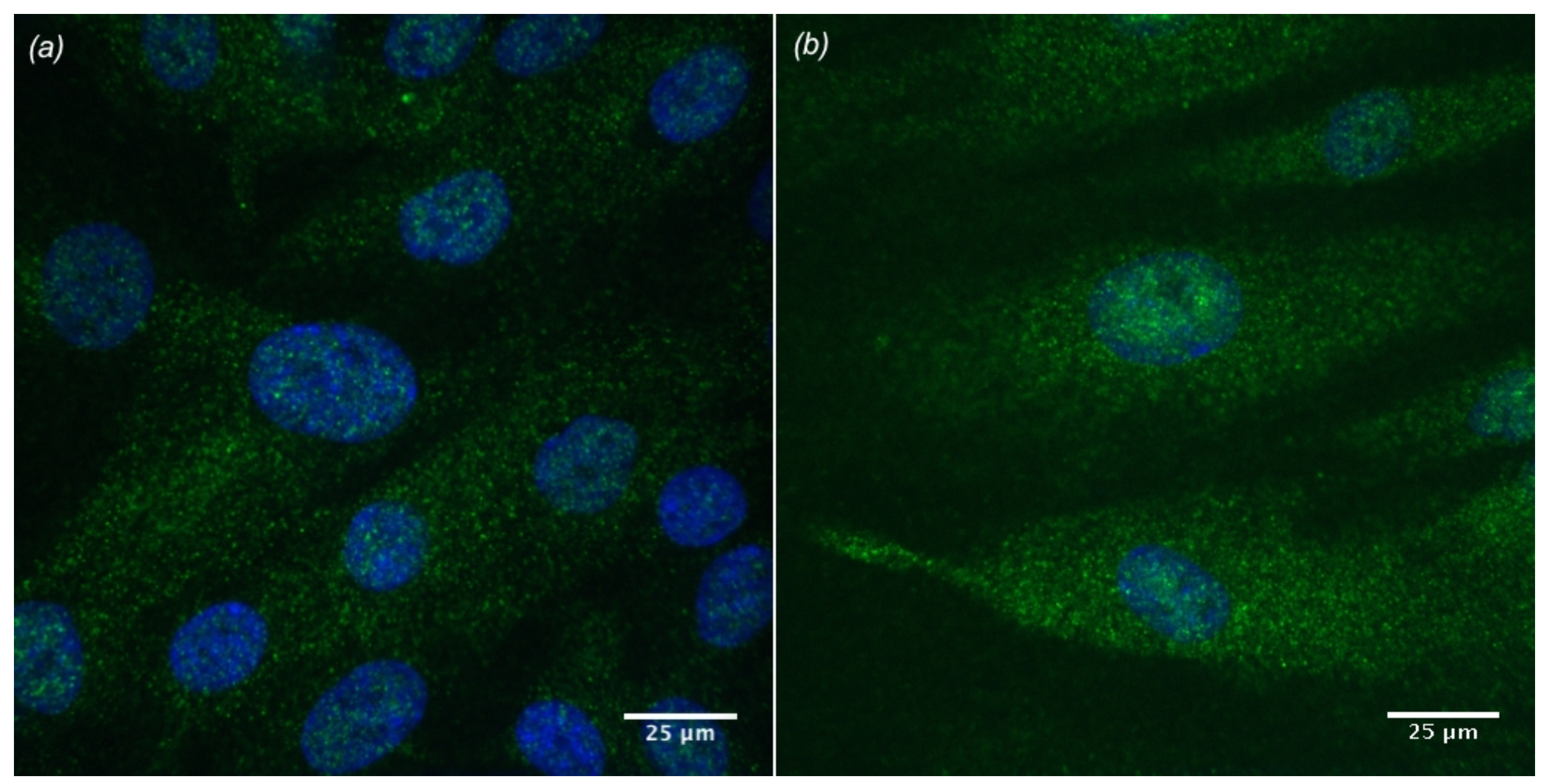


Table S1. Viability of NHDF treated for 24 heures with ethyl acetate natural extracts at $10 \mu \mathrm{g} / \mathrm{mL}, 1 \mu \mathrm{g} / \mathrm{mL}$ and $0,1 \mu \mathrm{g} / \mathrm{mL}$ in DMSO.

\begin{tabular}{|c|c|c|c|c|c|c|c|}
\hline $\begin{array}{l}\text { Extracts } \\
\text { SNB n }\end{array}$ & $\begin{array}{l}\% \text { Viability at } \\
10 \mu \mathrm{g} / \mathrm{mL}\end{array}$ & $\begin{array}{l}\% \text { Viability at } \\
1 \mu \mathrm{g} / \mathrm{mL}\end{array}$ & $\begin{array}{l}\% \text { Viability at } \\
0,1 \mu \mathrm{g} / \mathrm{mL}\end{array}$ & $\begin{array}{l}\text { Extracts } \\
\text { SNB } n^{\circ}\end{array}$ & $\begin{array}{l}\% \text { Viability at } \\
10 \mu \mathrm{g} / \mathrm{mL}\end{array}$ & $\begin{array}{l}\% \text { Viability at } \\
1 \mu \mathrm{g} / \mathrm{mL}\end{array}$ & $\begin{array}{l}\% \text { Viability at } \\
0,1 \mu \mathrm{g} / \mathrm{mL}\end{array}$ \\
\hline CN1 & 10,3 & 65,1 & 94,4 & CN111 & 11,3 & 995,6 & 91,4 \\
\hline CN10 & 10,5 & 62,1 & 90,1 & CN112 & 9,9 & 89,0 & 91,5 \\
\hline CN100 & 79,4 & 92,8 & 98,3 & CN16 & 89,1 & 94,0 & 90,8 \\
\hline CN11 & 10,6 & 58,5 & 86,2 & CN17 & 88,6 & 92,3 & 90,4 \\
\hline CN13 & 54,5 & 91,0 & 94,8 & CN29 & 44,9 & 86,8 & 85,4 \\
\hline CN14 & 10,6 & 55,9 & 91,6 & CN37B & 106,6 & 92,4 & 92,9 \\
\hline CN15 & $<5,0$ & 91,0 & 92,7 & CN55 & 9,6 & 86,4 & 93,4 \\
\hline CN2 & 10,7 & 92,1 & 94,2 & CN56 & 18,4 & 94,2 & 94,1 \\
\hline CN20 & 10,1 & 86,6 & 93,2 & CN57 & 11,1 & 79,7 & 91,9 \\
\hline CN27 & 98,4 & 93,0 & 92,6 & CN60 & 28,2 & 97,8 & 96,7 \\
\hline CN28 & 98,3 & 97,4 & 91,3 & CN60bis & 9,9 & 90,0 & 89,6 \\
\hline CN3 & 10,5 & 59,1 & 90,2 & CN65 & 19,3 & 89,4 & 88,7 \\
\hline $\mathrm{CN} 4$ & 13,1 & 90,5 & 88,9 & CN70 & $<5,0$ & ND & 87,0 \\
\hline CN52 & 112,2 & 91,6 & 89,3 & CN36A & 129,7 & ND & 89,0 \\
\hline CN61 & 96,7 & 88,7 & 89,1 & CN71 & 11,1 & 120,0 & 102,3 \\
\hline CN69 & 15,2 & 86,6 & 90,4 & CN72 & 10,0 & 115,6 & 92,9 \\
\hline CN78 & 49,5 & 93,3 & 89,2 & CN73 & 50,6 & 107,1 & 93,9 \\
\hline CN8 & 73,1 & 93,8 & 90,3 & CN74 & 14,0 & 97,5 & 95,4 \\
\hline CN88 & 71,6 & 88,4 & 89,9 & CN 82 & 80,3 & 92,7 & 92,9 \\
\hline CN89 & $<5,0$ & 92,7 & 92,5 & CN84 & 16,9 & 87,4 & 88,4 \\
\hline CN90 & 72,9 & 92,0 & 93,8 & CN85 & 34,9 & 83,5 & 84,1 \\
\hline CN91 & 92,0 & 97,3 & 91,3 & CN87 & 11,9 & 83,6 & 90,8 \\
\hline CN92 & 13,4 & 94,4 & 96,2 & GSS11 & 28,1 & 85,0 & 87,2 \\
\hline CN94 & 101,1 & 96,1 & 92,3 & GSS15 & 49,5 & 89,2 & 83,8 \\
\hline CN95 & 102,8 & 96,4 & 92,2 & GTC0202 & 12,7 & 88,2 & 88,5 \\
\hline CN96 & 9,7 & 97,3 & 91,5 & GTC2401 & 21,5 & 88,5 & 88,4 \\
\hline CN97 & 23,6 & 99,9 & 100,2 & GTC2701 & 18,3 & 89,8 & 87,3 \\
\hline CN98 & 64,9 & 90,2 & 88,9 & GTC2809 & 10,1 & 93,8 & 93,3 \\
\hline CN99 & 100,0 & 91,2 & 88,2 & CN75 & 10,8 & 91,9 & 101,2 \\
\hline CN102 & 9,8 & 90,2 & 90,5 & CN76 & 98,3 & 101,3 & 97,8 \\
\hline CN103 & 9,9 & 89,4 & 91,3 & GTC2810 & 28,7 & 85,7 & 91,3 \\
\hline CN104 & 100,5 & 90,4 & 87,1 & GTC2822 & 23,9 & 89,0 & 91,2 \\
\hline CN109 & 10,3 & 93,7 & 91,8 & GTC3002 & 63,4 & 92,4 & 92,5 \\
\hline CN110 & 15,2 & 92,4 & 88,7 & GSS07 & 11,0 & 91,7 & 91,6 \\
\hline LD2.10.2 & 51,9 & 84,5 & 97,7 & GSS04 & 49,9 & 91,8 & 86,8 \\
\hline LD2.13 & 16,1 & 89,5 & 87,5 & LD8.9 & 30,0 & 96,0 & 96,0 \\
\hline LD3.4 & 30,1 & 89,3 & 94,6 & CN67 & 84,7 & 95,1 & 93,3 \\
\hline LD6.5.2 & 17,4 & 87,5 & 98,6 & CN68 & $<5,0$ & ND & ND \\
\hline LD7.1 & 9,9 & 91,6 & 98,3 & Levure rose & $<5,0$ & 74,4 & 77,9 \\
\hline
\end{tabular}




\begin{tabular}{llll||llll} 
LD8.6 & 9,8 & 102,4 & 108,2 & CN113 & 10,1 & 79,5 & 81,6 \\
\hline CN79 & 100,9 & 82,5 & 85,3 & CN64 & 10,1 & 92,9 & 89,0 \\
\hline LD9.2 & $<5,0$ & 88,7 & 89,1 & CN66 & 98,6 & 90,0 & 92,3 \\
\hline CN59 & 12,7 & 86,5 & 92,4 & CN54 & 90,4 & 89,9 & 90,2 \\
\hline CN62 & 39,2 & 93,6 & 88,0 & & & & \\
\hline
\end{tabular}

$N D=$ Not determined . 
Table S2. Reproducibility expressed as Relative Standard Deviation (RSD) between triplicates of the same sample, and between biological duplicates of the same treatment.

\begin{tabular}{|c|c|c|c|c|c|}
\hline \multicolumn{3}{|c|}{ Reproducibility between triplicates } & \multicolumn{3}{|c|}{ Reproducibility: between biological duplicates } \\
\hline $\begin{array}{l}\text { Extracts } \\
\text { SNB n }\end{array}$ & $\begin{array}{l}\text { RSD for AGEs } \\
\text { measurement } \\
(\%)\end{array}$ & $\begin{array}{l}\text { RSD for } \\
\text { Pentosidine } \\
\text { measurement } \\
(\%)\end{array}$ & $\begin{array}{l}\text { Extracts } \\
\text { SNB } n^{\circ}\end{array}$ & $\begin{array}{l}\text { RSD for AGEs } \\
\text { measurement } \\
(\%)\end{array}$ & $\begin{array}{l}\text { RSD for } \\
\text { Pentosidine } \\
\text { measurement } \\
(\%)\end{array}$ \\
\hline Control & 4.0 & 2.4 & Control & 12.0 & 2.9 \\
\hline CN59 & 4.7 & 2.2 & CN59 & 3.9 & 1.5 \\
\hline CN79 & 3.7 & 3.8 & CN79 & 3.7 & 4.2 \\
\hline CN76 & 15.3 & 6.5 & CN76 & 16.5 & 6.1 \\
\hline GSS04 & 4.5 & 4.6 & GSS04 & 3.5 & 3.1 \\
\hline CN75 & 2.8 & 0.2 & CN75 & 4.4 & 0.1 \\
\hline CN67 & 5.5 & 2.8 & CN67 & 5.6 & 2.4 \\
\hline LD 9.2 & 4.1 & 19.3 & LD 9.2 & 6.4 & 16.4 \\
\hline GSS07 & 1.5 & 0.7 & GSS07 & 2.4 & 4.4 \\
\hline CN66 & 2.0 & 3.4 & CN66 & 4.1 & 3.1 \\
\hline CN85 & 11.8 & 0.4 & CN85 & 7.5 & 3.3 \\
\hline CN55 & 2.7 & 1.5 & CN55 & 10.7 & 9.0 \\
\hline CN1 & 2.4 & 1.6 & CN1 & 1.8 & 1.3 \\
\hline CN102 & 7.9 & 2.6 & CN102 & 5.6 & 2.1 \\
\hline CN69 & 2.3 & 0.5 & CN69 & 3.6 & 4.1 \\
\hline CN27 & 6.4 & 2.5 & CN27 & 5.6 & 12.6 \\
\hline CN13 & 9.0 & 0.5 & CN13 & 6.9 & 2.4 \\
\hline CN20 & 5.9 & 1.6 & CN20 & 7.0 & 6.5 \\
\hline GTC2810 & 3.5 & 0.8 & GTC2810 & 9.8 & 1.2 \\
\hline CN10 & 4.5 & 8.5 & CN10 & 18.3 & 6.13 \\
\hline CN104 & 3.3 & 1.4 & CN104 & 4.32 & 1.1 \\
\hline CN98 & 6.1 & 5.1 & CN98 & 14.6 & 10.6 \\
\hline CN54 & 6.7 & 0.6 & CN54 & 9.3 & 2.3 \\
\hline GTC2701 & 1.0 & 0.9 & GTC2701 & 4.7 & 2.1 \\
\hline $\begin{array}{l}\text { Amino- } \\
\text { guanidine }\end{array}$ & 3.9 & 1.2 & $\begin{array}{l}\text { Amino- } \\
\text { guanidine }\end{array}$ & 4.9 & 8.6 \\
\hline
\end{tabular}


Overexpression of AGER-1 in fibroblasts treated with extracts SNB-GSS07, SNB-GSS04 and SNB-CN13 at $1 \mu \mathrm{g} / \mathrm{mL}$ during 24 hours.

\section{Protocol:}

Cells were collected and solubilized in RIPA lysis buffer. Protein concentrations were determined using the BCA method. Equal amount of proteins $(26 \mu \mathrm{g})$ were separated on SDS-PAGE and transferred on $0.45 \mu \mathrm{m}$ PVDF membranes. The primary antibodies used were: monoclonal anti-vinculin antibody produced in mouse (Sigma-Aldrich \#V9131), anti-DDOST (AGER-1) antibody produced in rabbit (Sigma-Aldrich \#D6820). The following secondary antibodies were used: anti-mouse IRDye $800 \mathrm{CW}$ and anti-rabbit IRDye 680LT (LI-COR). Membranes were scanned with an Odyssey Imaging System (LICOR). Quantification was performed using ImageJ software. Unnecessary lanes were cut off and demarcated using black line in the following figure.

$\underline{\text { Results: }}$

A

SNB-GSS07 SNB-GSS04 SNB-CN13 Control

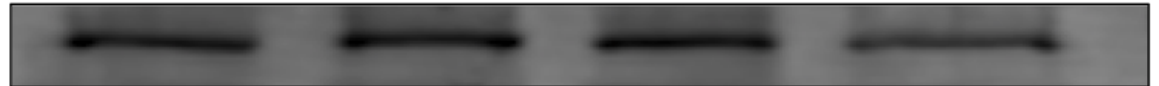

AGER-1

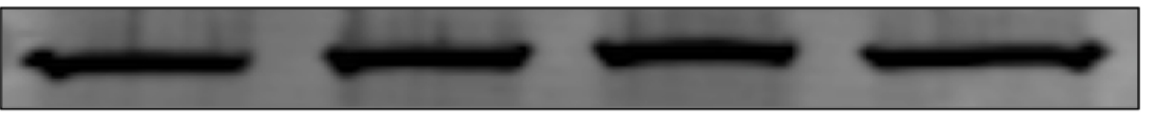

Vinculine

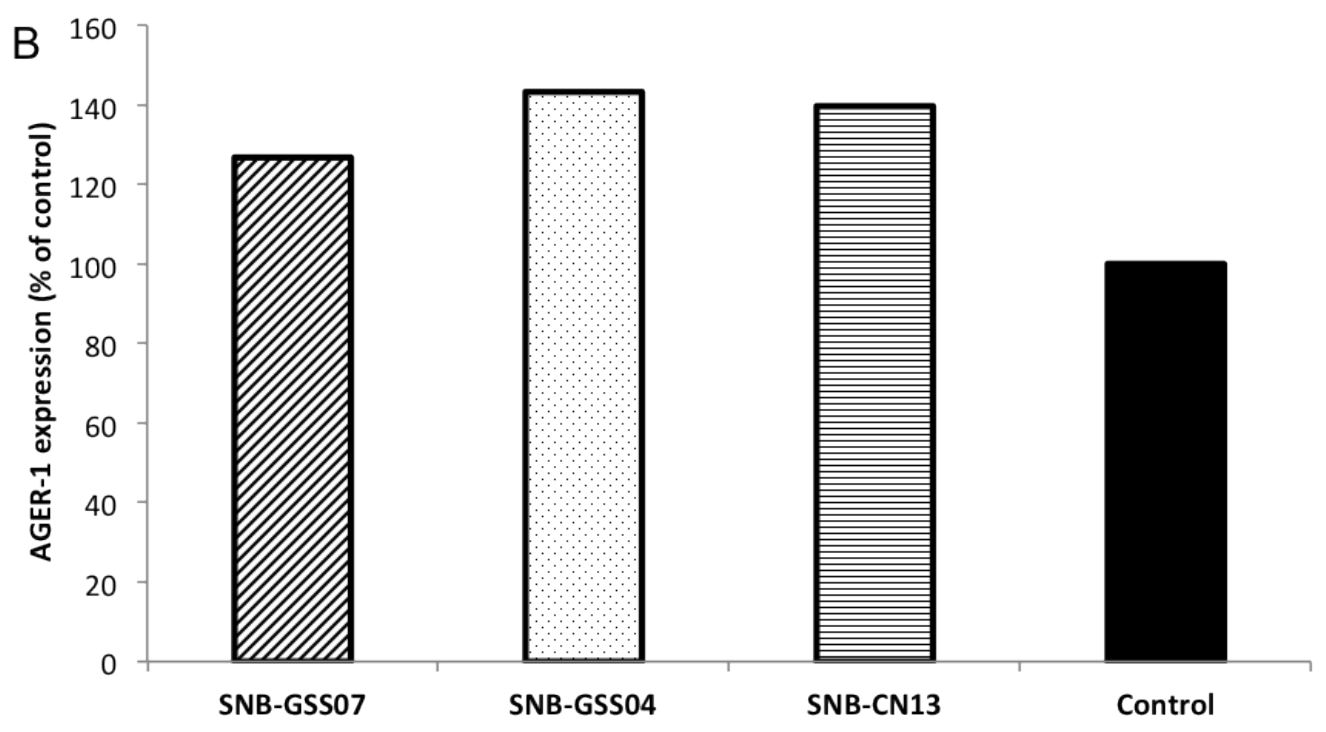

Figure S1. Overexpression of AGER-1 in fibroblasts treated with extracts SNB-GSS07, SNB-GSS0 4 and SNB-CN13 at $1 \mu \mathrm{g} / \mathrm{mL}$ during 24 hours. (A) Total protein extracts of treated and control fibroblasts were analysed by western blotting using anti-AGER-1 antibody. Vinculin was uses as loading control. (B) Quantification of AGER-1 expression in cells (date shown in A) and expressed as percentage of control. 